\title{
Vortices in rotating and stratified flows: aspect ratio and sustainability
}

\author{
O. Aubert ${ }^{1, a}$, M. Le Bars ${ }^{1}$, P. Le Gal ${ }^{1}$, and P. S. Marcus ${ }^{2}$ \\ ${ }^{1}$ Institut de Recherche sur les Phénomènes Hors Equilibre, UMR 7342, CNRS and Aix-Marseille Université, \\ 49 rue F. Joliot Curie, 13384 Marseille, Cédex 13, France \\ ${ }^{2}$ Department of Mechanical Engineering, University of California, Berkeley, CA 94720, USA
}

\begin{abstract}
In linear stratifications, vortices have a typical flat shape that appears to be not only a compromise between the rotation and the stratification of the background flow through their Coriolis parameter $f$ and buoyancy frequency $\bar{N}$, but also involves the buoyancy frequency $N_{c}$ within the vortices and their Rossby number $R o$. We derive an analytical solution for the self-similar ellipsoidal shape of the vortices and the law for their aspect ratio. From this law, we show that long-lived vortices must necessary be either weakly stratified anticyclones or superstratified cyclones (which is less likely to occur). These predictions are experimentally and numerically verified and agree with published measurements for Jovian vortices and ocean meddies. This approach can be applied to a gaussian stratification to give good insights of the shape of vortices in protoplanetary disks and their sustainability.
\end{abstract}

\section{Introduction}

The scenario of planetesimal formation in protoplanetary disks proposed by Barge \& Sommeria (1995) [1] is based on the trapping of solid dust particles in persistent anticyclonic vortices. Rotation and a symmetric Gaussian stratification with respect to the equatorial plane are two main ingredients of protoplanetary disks. There is now extensive numerical proof that anticyclonic long-lived coherent structures effectively trap particles in rotating flows, and numerical scenarios that also take into account the stratification of the disk [2]. These structures are thus of main importance for the understanding of planet formation.

Rotation and stratification have antagonistic effects: while rotation homogeneizes the flow along the axis of rotation, stratification on the contrary limits vertical motions. The amplitude of these two effects are measured through the Coriolis parameter $f=2 \Omega \sin \phi$ and the buoyancy frequency $\bar{N}=\sqrt{-g / \rho d \bar{\rho} / d z}$. Here $\Omega$ is the rotation rate of the planet (or the disk) weighted by the latitude $\phi, g$ is gravity, $\bar{\rho}$ is the fluid density and $z$ is the vertical coordinate. We investigate this competition using experiments in a rotating and stratified layer of fluid. This allows us to determine a generic law for the aspect ratio of vortices in rotating and stratified fluids that has been published in Aubert et al. (2012) [3] and Hassanzadeh et al. (2012) [4]. This law applies to vortices from the lab to natural

\footnotetext{
ae-mail: aubert@irphe.univ-mrs.fr
} 


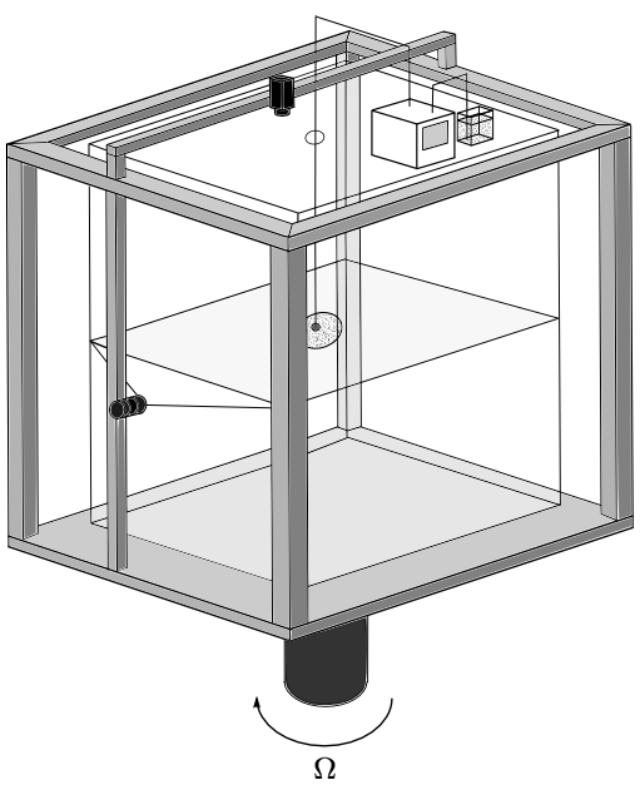

Figure 1. Schematic representation of the experiment tank of dimensions $50 \times 50 \times 70 \mathrm{~cm}^{3}$. This setup has Coriolis parameters ranging from 1 to $7 \mathrm{rad} / \mathrm{s}$, and buoyancy frequencies $\bar{N}$ from 0.6 to $2.4 \mathrm{rad} / \mathrm{s}$. While the tank is rotating and once the linearly stratified fluid inside has reached solid-body rotation, fluid at mid-density and seeded with PIV particle and dye is injected by a pump in the middle of the stratification on the axis of rotation. As the fluid is injected on the axis of rotation of the tank, its rotation rate in the rotating frame of reference is initially $\Omega_{v}=\Omega$, imposing an initial Rossby number $R o(0)=\Omega_{v}(0) / 2 \Omega=1 / 2$.

environments such as the vortices in the Jovian atmosphere or the Meddies in the Atlantic Ocean and could be of great interest to predict the shape of vortices in proto-planetary disks.

\section{Experimental setup}

Similarly to the experiments of [5] and [6], we re-create in the laboratory the two main ingredients of the considered problem, i.e., rotation and stratification, by filling a rectangular transparent tank with linearly stratified salty water, that is fixed on a rotating table. After filling the tank with the stratified water, it is progressively put into motion until all the water is in solid-body rotation.

As shown in Figure 1, the top of the tank is equipped with a pump and an injector that is fixed on the axis of rotation and goes down into the middle of the stratification. Using these devices, we either briefly inject a fixed volume of homogeneous fluid whose density is the same as the one of the background at the height of injection, or continuously inject homogeneous fluid at a fixed rate. The homogeneous injected fluid spreads on the horizontal plane corresponding to its density and the Coriolis force rapidly deflects the fluid particles in an anticyclonic motion. The brief injection creates vortices whose Rossby number Ro quickly decreases until they reach equilibrium, whereas the continuous injection is much smoother and allows us to maintain higher $|R o|$ that decrease much more slowly.

Using a horizontal laser sheet and a video camera placed at the top of the tank, we extract the velocity profiles in the horizontal $(z=0)$ plane at the center of the vortex as seen in Figure 2, using DPIVsoft [7]. A vertical laser sheet seen from a side camera allows to extract the contour of the vortex in the vertical plane and its vertical aspect ratio. 

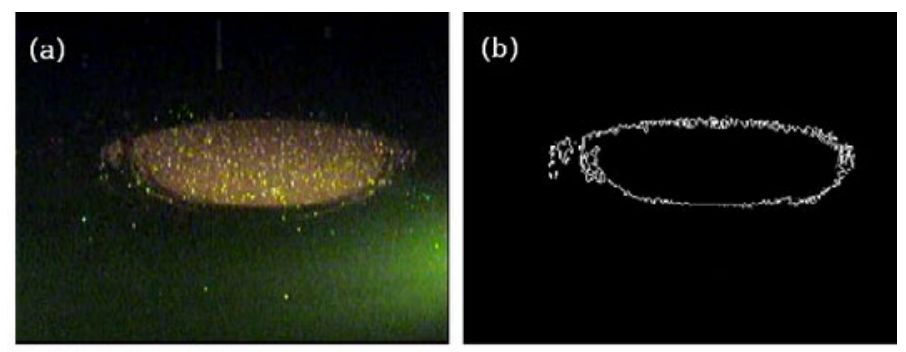

Figure 2. Experimentally generated anticyclonic vortex with $\bar{N}=2.3$ $\mathrm{rad} / \mathrm{s}, f=2 \mathrm{rad} / \mathrm{s}$ : (a) side view; (b) extracted contour by image processing to determine the aspect ratio $\alpha=0.3$ (thickness $3.8 \mathrm{~cm}$ and diameter $12.6 \mathrm{~cm}$ ); (c) top view; (d)
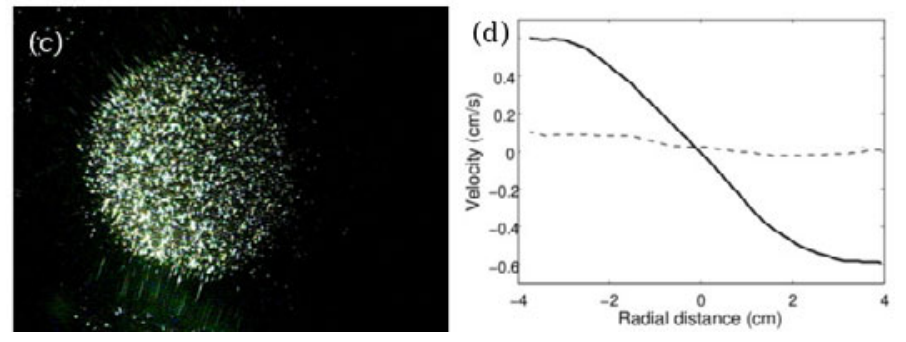
velocity profile in an horizontal plane obtained by PIV post-processing (we measure a velocity field from sequences of top views like (c), from which velocity profiles are extracted along the diameter of the vortex).

\section{The universal aspect ratio of vortices in rotating stratified fluids}

\subsection{Theoretical derivation}

Vortices in equilibrium in a rotating stratified fluid are in cyclo-geostrophic balance in the horizontal plane and in hydrostatic balance in the vertical direction. These balances reduce to the gradient-wind equation (eq. (1)) where $v$ is the azimuthal velocity and $\rho^{\prime}$ is the density anomaly with the background fluid:

$$
\frac{\partial v}{\partial z}\left(\frac{2 v}{r}+f\right)=-\frac{g}{\rho_{0}} \frac{\partial \rho^{\prime}}{\partial r}
$$

A scaling analysis of this equation as in [4] shows that the vertical aspect ratio of vortices in rotating stratified fluids not only depends on the parameters $\bar{N}$ and $f$ of the environment but also on intrinsic properties such as the vortex velocity through the Rossby number $R o=\Omega_{v} / f$, where $\Omega_{v}$ is the rotation rate at the center of the vortex, and the stratification of its core through the corresponding internal buoyancy frequency $N_{c}$. Note that natural vortices are barely homogeneous and one can define an inner stratification. The vertical aspect ratio $\alpha=H / L$, where $H$ and $L$ are the vertical and horizontal length scales of the vortex respectively, is given by

$$
\alpha=\left(\frac{R o(1+R o)}{N_{c}^{2}-\bar{N}^{2}}\right)^{1 / 2} f .
$$

This law brings a new understanding on the asymmetry between cyclones and anticyclones in nature. Indeed, the solutions are cyclones $(R o>0)$ with a super-stratified interior $\left(N_{c}>\bar{N}\right)$ and weak anticyclones $(-1<R o<0)$ with a less stratified interior than the background fluid $\left(N_{c}<\bar{N}\right)$, and one can understand that it is easier to locally mix some fluid to create a patch with a weaker stratification than the background, rather than to locally super-stratify a patch of fluid. It agrees well with published measurements on Jovians vortices and Meddies as shown in [3].

\subsection{Modeling and vertical shape}

After analyzing experimentally measured velocity profiles, we propose a model (3) for the azimuthal velocity $v$ in the rotating frame of reference corresponding to a solid-body rotation of the core of the 


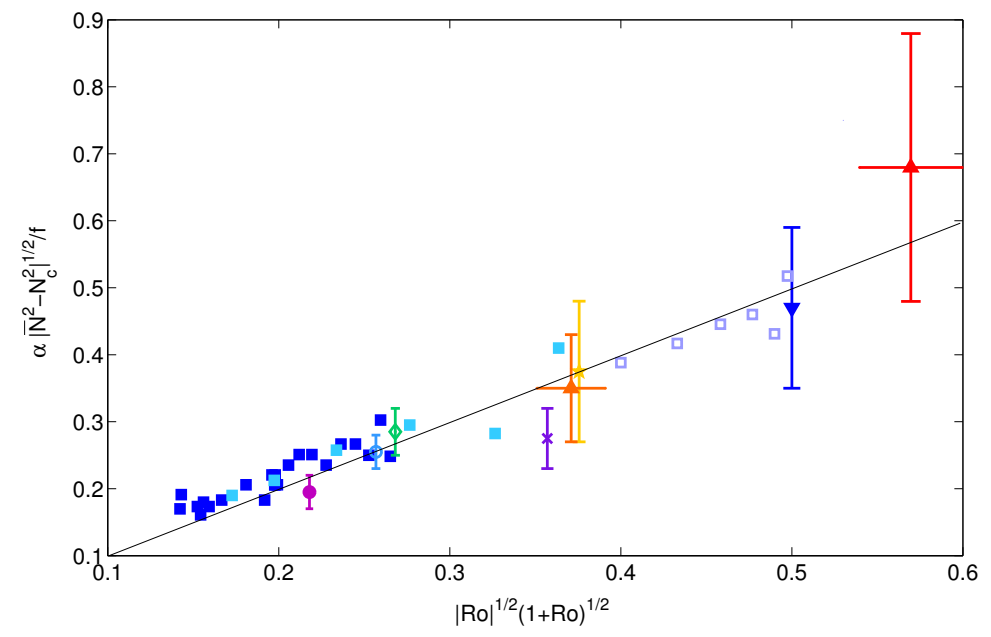

Figure 3. Tests of our theoretical law (2) (straight line) with our freely decaying vortex experiments (匹) and with our sustained vortex experiments ( $\square$ ); with Bush \& Woods' experiments ( $\mathbf{v})$; with Jupiter's Great Red Spot and Oval BA (४); and with meddies Sharon $(\times)$, Bobby $(\star)$, Hyperion $(\circ)$, Ceres $(\bullet)$ and Encelade $(\diamond)$. Details and references can be found in [3].

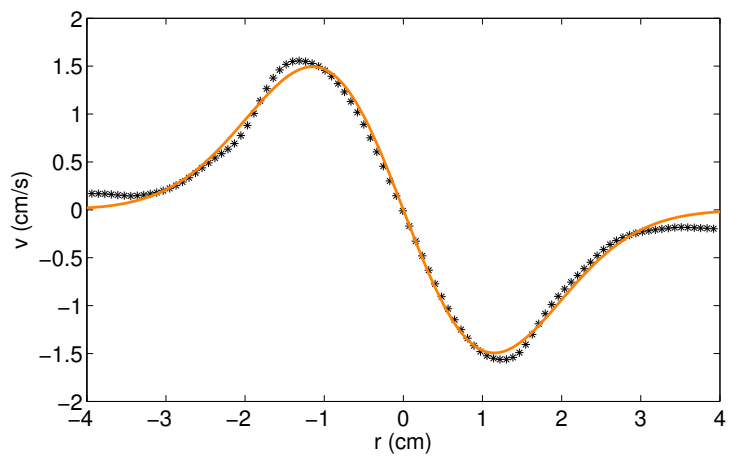

Figure 4. Theoretical $v$ profile given by the model (3) (-) compared with the experimentally obtained velocity profiles $(\star)$ in the centered horizontal plane $(z=0)$. Ro and $L$ being the two parameters of the model in this plane, they are given by the best fit of the model with the experimental curve: $\bar{N}=1.6 \mathrm{rad} / \mathrm{s}, N_{c}=0 \mathrm{rad} / \mathrm{s}$, $f=6.8 \mathrm{rad} / \mathrm{s}$ and the measured parameters are $R o=0.17$ and $L=2.58 \mathrm{~cm}$.

vortex and an exponential decay in the radial and vertical directions to match the zero velocity of the background. This decay occurs on the characteristic spatial scales $L$ and $H$. A fit of this model is given in Figure 4. The model for the density anomaly $\rho^{\prime}$ is determined so as to satisfy the gradient-wind equation (1).

$$
v(r, z)=f \operatorname{Ror} e^{-(r / L)^{2}-(z / H)^{2}}
$$

By comparing the position of the horizontal length scale $r=L$ in the model for the velocity profile (3) and the position of the frontier of the dye injected in the experiments, we define the radius of the vortex $R$ by the position of the maximum of the radial gradient of the velocity in the horizontal centered plane, i.e., $R=\max (\partial v / \partial r, z=0)$, which corresponds to the inflexion point in the velocity profile. Applying the same reasoning to the density anomaly $\rho^{\prime}$, we define the half-thickness of the vortex to be located at the maximum of the vertical density gradient on the axis of rotation, i.e. $Z=\max (\partial v / \partial z, r=0)$. This leads to $R=\sqrt{3 / 2} L$ and $Z=\sqrt{3 / 2} H$. The elliptic contour of the vortex in the vertical plane with this model is thus given by

$$
\left(\frac{r}{L}\right)^{2}+\left(\frac{z}{H}\right)^{2}=\frac{3}{2}
$$




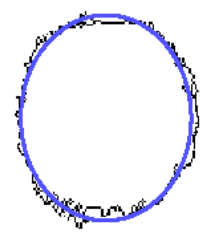

Ro $=-0.08$

$\mathrm{t} / \mathrm{T}=276$

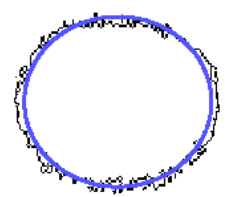

Ro $=-0.046$

$\mathrm{t} / \mathrm{T}=568.2$

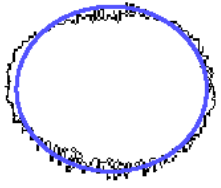

Ro $=-0.041$

$\mathrm{t} / \mathrm{T}=\mathbf{8 6 0 . 4}$

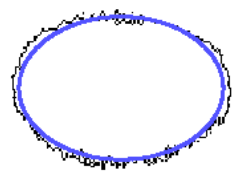

Ro $=-0.027$

$\mathrm{t} / \mathrm{T}=1452.9$

Figure 5. Image-processed side-view of a vortex boundary (white) at different times $t$ with $f=6.8 \mathrm{rad} / \mathrm{s}$ and $\bar{N}=1.6 \mathrm{rad} / \mathrm{s}$ held fixed. $|R o|$ decreases in time. Also shown are the theoretical boundaries (blue) of the model vortices at each time. $T=4 \pi / f$ is the period of the rotating turntable.

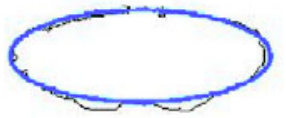

$\mathrm{Ro}=-0.45$

$\mathrm{t} / \mathrm{T}=77$

$\mathrm{V}=87 \mathrm{~mL}$

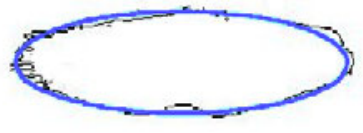

Ro $=-0.25$

$\mathrm{t} / \mathrm{T}=139$

$\mathrm{V}=167 \mathrm{~mL}$

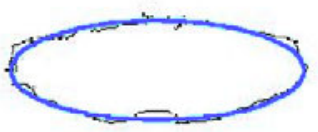

$\mathrm{Ro}=-0.35$

$\mathrm{t} / \mathrm{T}=108$

$\mathrm{V}=127 \mathrm{~mL}$

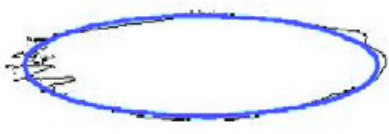

Ro $=-0.2$

$\mathrm{t} / \mathrm{T}=155$

$\mathrm{V}=187 \mathrm{~mL}$
Figure 6. Image-processed side-view boundaries of a vortex sustained with continuous injection at different times. The flow has $f=1.62 \mathrm{rad} / \mathrm{s}$, $\bar{N}=2.3 \mathrm{rad} / \mathrm{s}$, and an injection rate of $10 \mathrm{~mL} / \mathrm{min}$. Also shown are the theoretical boundaries of the model vortices. Line colors are as in figure 5 . Unlike the vortex in figure 5, the vortex volume $V$ changes in time. $R o$, $V$, and time $t$ are given for each image.

where $H$ and $L$ change through time, following the law for the aspect ratio (2) and the conservation of volume.

\section{Insights of vortices in proto-planetary diks}

Following the approach described in the previous section, we started to look at the shape and aspect ratio of vortices in a Gaussian stratification. In Figure 4 we give some shapes one would obtain in our experiments if the background fluid had a Gaussian profile of stratification, for a vortex injected with a given density. It appears that the most coherent shapes are obtained for vortices above and below the mid-plane, where the buoyancy frequency is at its maximum, and not in the mid-plane, where $\bar{N}=0$. To complete a study on the predominance and vertcal position of anticyclones in proto-planetary disks, one would have to add shear into these experiments.

\section{References}

[1] P. Barge \& J. Sommeria, Astronomy \& Astrophysics 295, L1 (1995) 


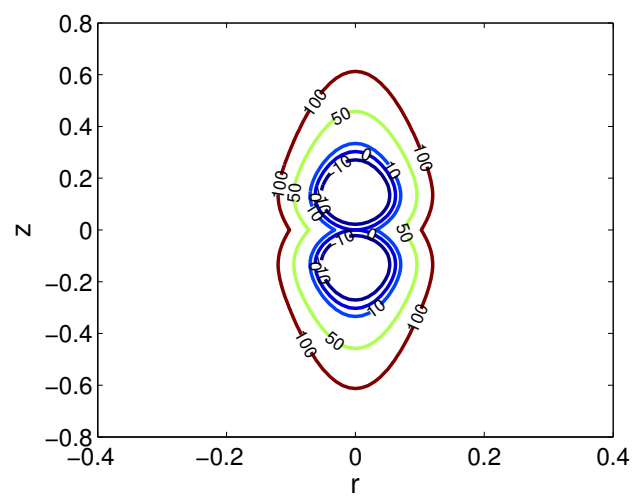

Figure 7. Contours of a vortex in a gaussian stratification with $\rho_{0}=1060 \mathrm{~kg} / \mathrm{m}^{3}$ in the mid-plane, for $\rho_{v}=1013$ $\mathrm{kg} / \mathrm{m}^{3}$ and different volumes.

[2] J. A. Barranco \& P. S. Marcus, Astrophysical Journal 623, 1157 (2005)

[3] O. Aubert, M. Le Bars, P. Le Gal \& P. S. Marcus, Journal of Fluid Mechanics 706, 34-45 (2012)

[4] P. Hassanzadeh, P. S. Marcus \& P. Le Gal, Journal of Fluid Mechanics 706, 46-57 (2012)

[5] R. W. GRiffiths \& P. Linden, Journal of Fluid Mechanics 105, 283-316 (1981)

[6] K. Hedstrom \& L. Armi, Journal of Fluid Mechanics 191, 535-556 (1988)

[7] P. Meunier \& T. Leweke, Experiments in Fluids 35, 408-421 (2003) 